\title{
$\underline{\text { Spectral Science Priorities for an Infrared Spectrometer with Interstellar Probe }}$
}

White Paper to be submitted to the 2023 - 2032 Planetary Science Decadal Survey, 2020

Caitlin Ahrens* (Arkansas Center for Space and Planetary Sciences, University of Arkansas, 332

North Arkansas Ave., Fayetteville, AR 72701; ca006@uark.edu)

Carey Lisse ${ }^{1}$, Michael Zemcov ${ }^{2}$, Kirby Runyon ${ }^{1}$

${ }^{1}$ Johns Hopkins University Applied Physics Laboratory; ${ }^{2}$ Rochester Institute of Technology; 


\section{Background}

The exploration of the outer solar system (especially the Kuiper Belt), heliosphere and beyond brings new opportunities to observe our local universe in different wavelengths, particularly the infrared spectrum. Observations in the infrared provide exciting and profound understandings of the structure of different objects, from planetary bodies to disk dust. However, there is a lack of studies involving an overall infrared spectral understanding of our solar system and beyond. This paper addresses the components and motivations to the infrared spectral exploration through the interdisciplinary use of an Interstellar Probe (see white paper by Brandt et al., 2020).

Mapping the infrared compositional universe can be accomplished in the coming decade by the Interstellar Probe by observing 1) compositional extent of ices and chemical boundaries, especially the amount of water ice; 2) solar irradiation interactions, especially for sublimation of ices or organic concentrations; 3) observing and characterizing the dust IR signatures with implications to zodiacal clouds and origins; 4) development of space-based instrumentation for the identification of such IR chemical characteristics.

The discussion is divided into three main sections, addressing fundamental questions as they pertain to the spectral significance of understanding our local universe:

-What are the key scientific questions that will be motivating the study of the infrared spectroscopy of various objects?

-What discoveries from previous missions have led us to these key questions?

-What progress can be made in the next decade to answer these questions?

What are the key scientific questions that will be driving forward the motivation to study the infrared spectroscopy of various objects?

\section{Planetary:}

-What is the role of water ice on the surface of outer solar system bodies and Kuiper Belt Objects (KBOs)? (Baragiola 2003; also see white paper by Runyon et al. 2020 for more on the KBO planetology research by an Interstellar Probe)

-What are the concentrations of various ices $\left(\mathrm{H}_{2} \mathrm{O}, \mathrm{CO}_{2}, \mathrm{CH}_{4}, \mathrm{~N}_{2}, \mathrm{NH}_{3}\right.$, etc.) across the outer solar system and beyond? 
-Is there an interaction of icy debris/dust with the KBOs?

-What is the extent of irradiation (and thus irradiated ices) on KBOs?

\section{Heliophysics:}

-What are the impacts of solar irradiation/tholin production on the KBO surfaces?

-What are the interactions of interstellar dust with our heliosphere? (Duley and Williams 1981; also see white paper by Szalay et al. 2020)

-Is there an observation of irradiated product variability (or gradient) across the Kuiper Belt? (Moore et al. 2004)

\section{Astrophysics:}

-What is the dust production and distribution of our universe? (Mathis 1990; Draine 2003)

-What are the large-scale distributions of dust in the zodiacal cloud and beyond?

-Is there a second Kuiper Belt dust cloud (as predicted by Stark and Kuchner 2009; Poppe 2016)?

\section{What discoveries from previous observations have led us to these key questions?}

\section{Planetary:}

The NASA New Horizons Pluto fly-by exhibited the extraordinary diversity of undiscovered worlds in the Kuiper Belt that can unlock secrets of the chemical evolution of our solar system, specially using the Ralph instrument, the visible-infrared multispectral imager at Pluto (Reuter et al. 2008). Ices at outer solar system moons and KBOs offer an interesting (and still complex) look at different chemical structures, irradiated products, and crystalline-amorphous structures possibly influencing the evolution of these objects.

An example of a still-unexplored KBO is Quaoar at 40-50 AU (Fraser and Brown 2010; Fraser et al. 2013a,b), where this dwarf planet lines up with the nose direction of the heliosphere and the mysterious "Ribbon" of energetic neutral atoms (McComas et al. 2009), which may be losing a methane atmosphere (Fraser et al. 2013a,b). Crystalline ice has also been detected on Quaoar, implying an active surface (or previously active) for the temperature-pressures of the water ice to remain stable (Jewitt and Luu 2004). Such ices have been compared to Pluto and Charon using the $1-2.5$ um near-infrared wavelength regime (Figure 1), but more detailed spectroscopy is still needed and at longer wavelengths for possible organic tholin compounds. 


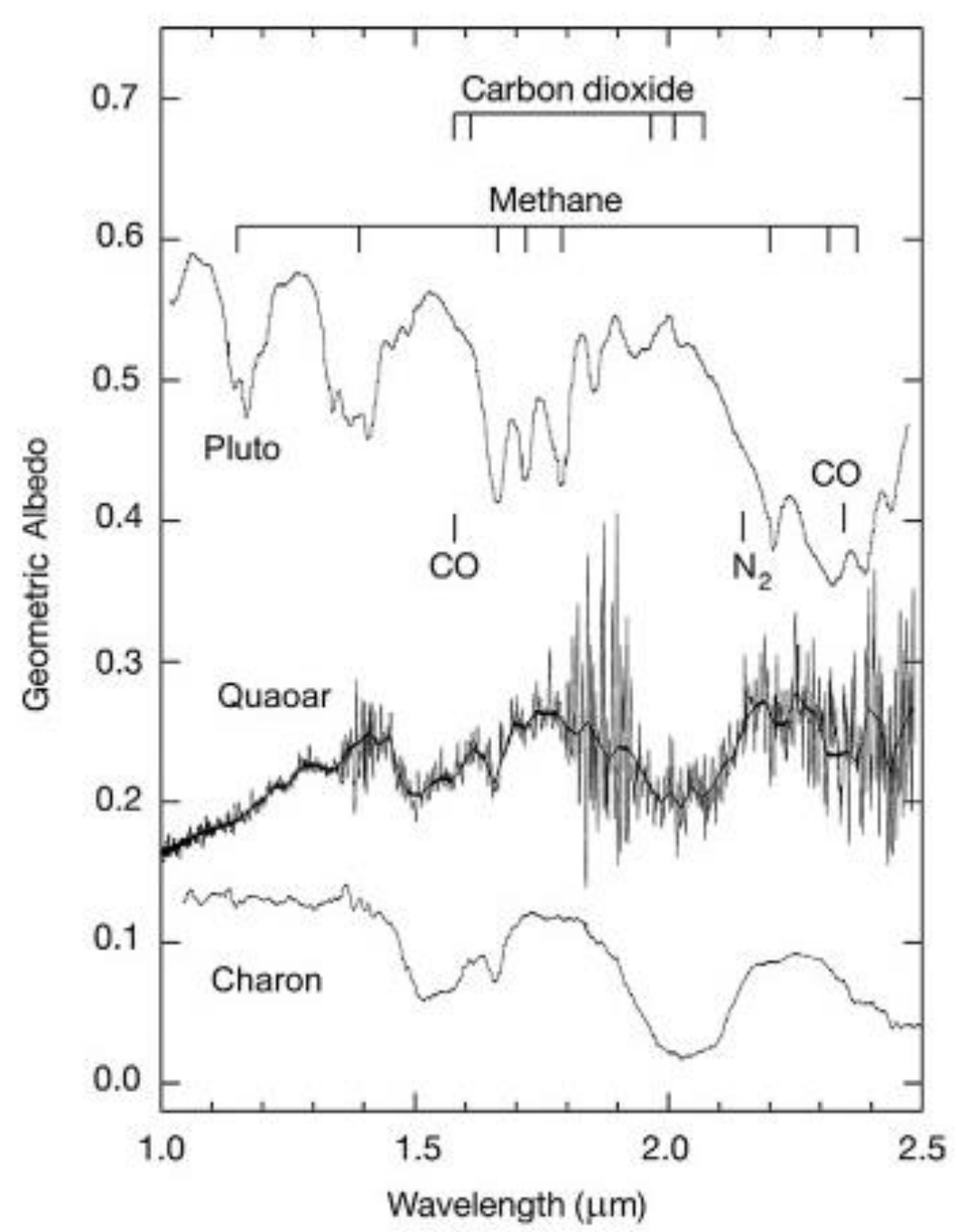

Figure 1: Near-infrared spectrum of Quaoar compared to near-infrared spectra of Pluto and Charon. The spectra of Quaoar and Charon are similar in that they exhibit three strong $\mathrm{H}_{2} \mathrm{O}$-ice absorption bands at $1.5,1.65$, and $2.0 \mu \mathrm{m}$, but no $\mathrm{CH}_{4}$-ice bands. The spectrum of Pluto exhibits strong $\mathrm{CH}_{4}$-ice bands. Image credit: Tegler (2014).

Traveling beyond the heliosphere, Interstellar Probe would provide an exceptional platform to observe the entire extent of the zodiacal cloud and to resolve a possible second Kuiper belt dust cloud isolated by the outer planets (Stark and Kuchner 2009; Poppe 2016; Poppe et al. 2020 White Paper). Near-Infrared (NIR) imagery can inform about the dust cloud density, while MidInfrared (MIR) cameras could provide thermal imaging photometry related to dust particle size and composition. Explorations for the presence of rings and dust clouds around otherwise discrete sources could be enabled by observing at high phase angles towards the Sun from $>200$ AU. For example, searches for debris clouds associated with the Haumea family collisional fragments, or the rings of the Centaur Chariklo, or dust emitted from bombardment off the six known bodies of the Pluto system (McNutt Jr. et al. 2019). 
Astrophysics:

Our circum-solar debris disk consists of the zodiacal cloud, which is formed from dust created by grinding debris from our Asteroid and Kuiper Belts and by dust spewed from comets. While the distribution of the asteroids and KBOs is relatively well studied, the dust production and evolution of such dust in the Kuiper Belt and beyond remains unclear. Circum-stellar debris disks are created by collisional processes between planetesimals and are therefore generally considered to provide fundamental insights into planetary formation and evolution (e.g., Lisse et al. 2012, 2017 and references therein). The dust distribution in the Kuiper Belt also provides important constraints on the total number of bodies in the belt, especially the smallest ones, and their dynamical collisional state.

The large-scale dust distribution beyond the zodiacal cloud is largely unknown because the observational vantage points in the infrared are all within this cloud. Several basic aspects of even the zodiacal cloud remain unexplained, which offers critical importance for continuous observations.

While ground-based (e.g., observations on Mauna Kea) and space-based telescopes (e.g., Spitzer Space Telescope, and the upcoming James Webb Space Telescope) have made important revelations to the distribution of ices and dust around exoplanetary systems in a range of wavelengths (Figure 2), such techniques can be applied to our own solar system and use our own solar system as an exoplanetary analog.

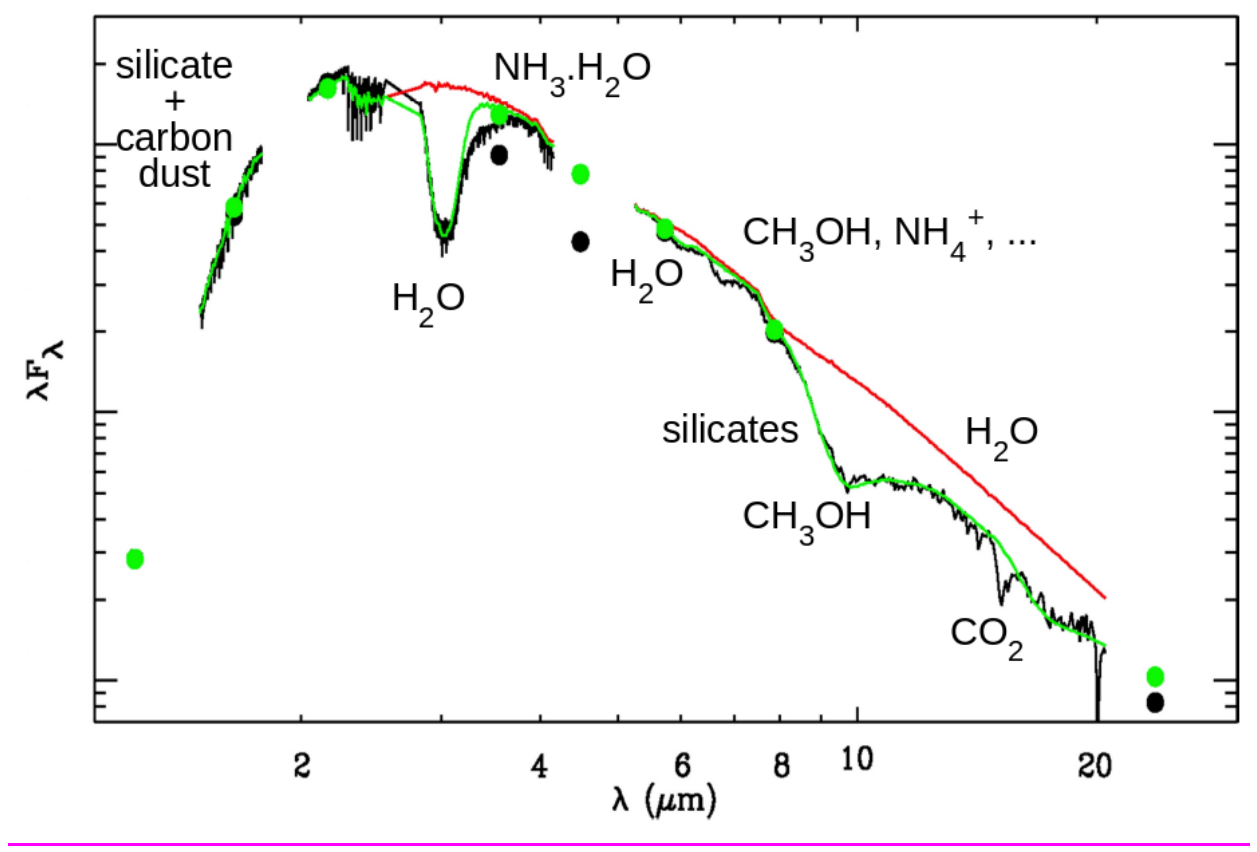

Figure 2: IR spectrum of a highly reddened star tracing silicate and carbon dust with icy mantles in a dense molecular cloud (black lines). It was observed with the Keck II telescope on Mauna Kea $(\lambda<4 \mu \mathrm{m})$ and the Spitzer Space Telescope $(\lambda>5 \mu \mathrm{m})$. The red and green lines are models 
used to extract the dust and ice signatures. Image credit:

https://www.ifa.hawaii.edu/research/ISM.shtml

\section{What progress can be made in the next decade to answer these questions?}

To supplement these observations, advances in modeling capabilities can further assist in IR chemistry and spectroscopy. Laboratory work for low temperature-low pressure studies have also improved to better understand the origin of ice grain condensation and irradiated products, such as tholins and other complex by-products, on planetary or cometary surfaces (McDonald et al. 1996; Yu et al. 2018).

Many of these questions are not exclusive to any one community alone and answering them will require an interdisciplinary and collaborative approach. A spectrometer onboard such an Interstellar Probe can observe several wavelengths for the purpose of researching multiple chemical species (as shown in Figure 3). For example, interpreting the IR spectroscopy of irradiated ice grains in the outer solar system requires knowledge of solar system surface processes (Planetary), space weathering and irradiation influences (Planetary and Heliophysics), and interstellar medium grain formations/dust-organic interactions (Astrophysics).

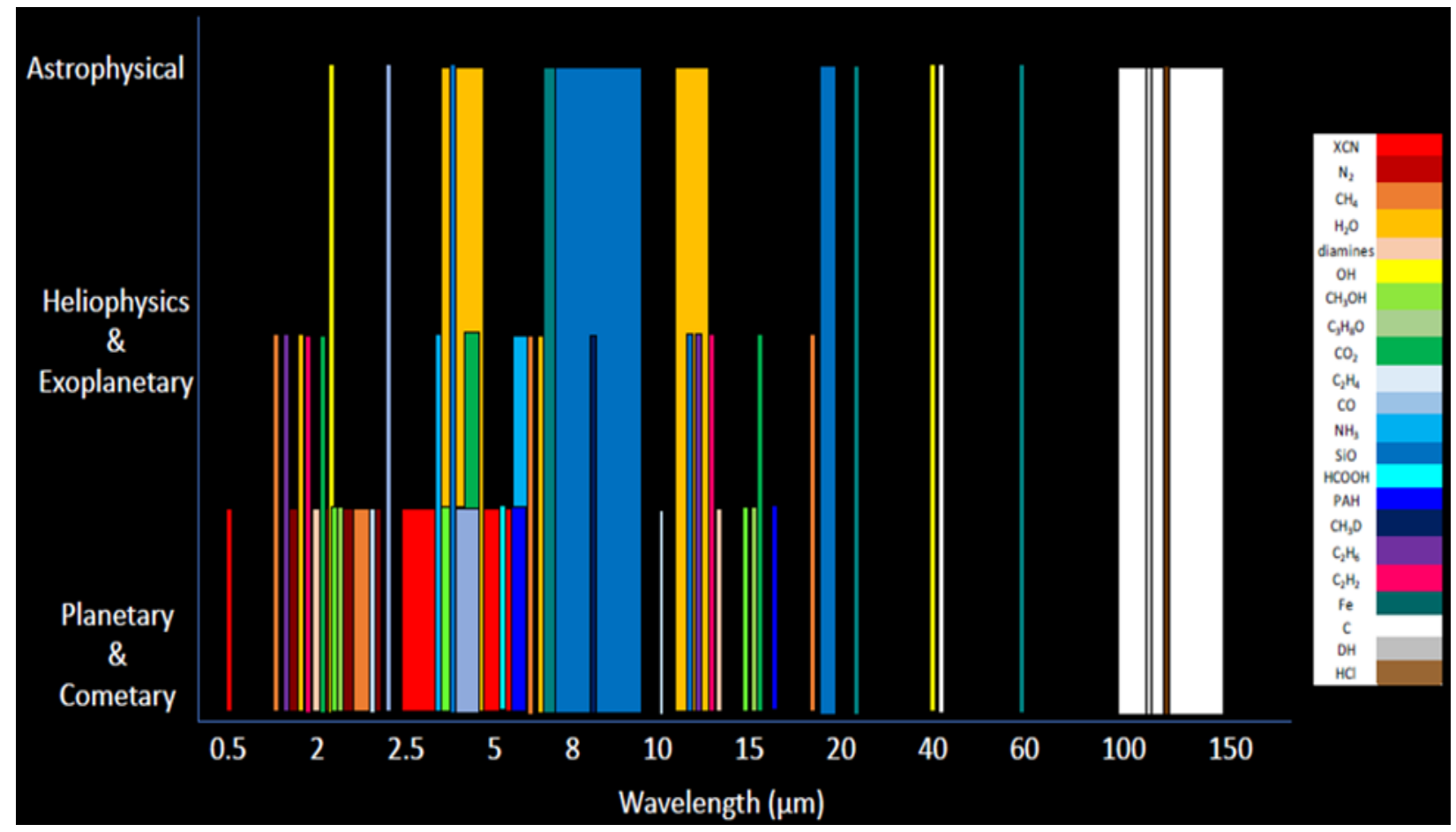

Figure 3: Diagram of potential chemical species in the solar system and beyond to observe with a multi-wavelength spectrometer on an Interstellar Probe, marking each chemical component as a priority for planetary, heliophysics/exoplanetary, and astrophysical. 


\section{The Next Step in Infrared Spectroscopy Exploration using the Interstellar Probe: Recommendations to NASA}

As missions to the outer planetary solar system (Voyagers) and beyond (New Horizons at Pluto), as well as astrophysical and solar missions (Parker Solar Probe, Spitzer, etc.) gave us insight to complex chemistries, the Interstellar Probe would give us an interdisciplinary vantage point to the breadth of infrared spectroscopy, not only by advancing technological capabilities for multiwavelength purposes, but enhancing our knowledge of the infrared universe.

Because none of the puzzling problems are likely to be resolved by near-Earth orbit telescopes, the case for venturing beyond the Kuiper Belt and beyond is strong. The overarching goal of a future Interstellar Probe infrared survey should be exploring multiple objects at various placements along the trajectory by identifying infrared volatiles and complex chemistries. As we have described in this report, the numerous, open scientific issues surrounding the infrared universe as a whole and specifics of debris dis dust and KBOs motivate calls for an infrared spectrometer onboard an Interstellar Probe.

\section{References}

Baragiola, R. (2003) Water ice on outer solar system surfaces: Basic properties and radiation effects. Planetary and Space Science, 51 (14-15), 953-961.

Brandt, P. et al. (2020) Interstellar Probe: Pushing the boundaries of space exploration. A white paper for the Solar \& Space Physics Decadal Survey 2023.

Draine, B. (2003) Interstellar dust grains. Annu. Rev. Astron. Astrophys. 41, 241-289.

Duley, W., and Williams, D. (1981) The infrared spectrum of interstellar dust: Surface functional groups on carbon. MNRAS, 196 (2), 269-274.

Fraser, W., Brown, M. (2010) Quaoar: A rock in the Kuiper belt. Astrophys. J., 714, 1547-1550.

Fraser et al. (2013a) The mass, orbit, and tidal evolution of the Quaoar-Weywot system. Icarus, 222, 357-363.

Fraser, W., et al. (2013b) Limits on Quaoar's atmosphere. Astrophys. J. Lett., 774, L18.

Jewitt, D., Luu, J. (2004) Crystalline water ice on the Kuiper belt object (50000) Quaoar. Nature, $432,731-733$.

Lisse, C., et al. (2012) Spitzer evidence for a Late-Heavy Bombardment and the formation of ureilites in n Corvi's Disk at 1 Gyr. Astrophys. J., 747, 93. 
Lisse, C., et al. (2017) Infrared spectroscopy of HR 4796A's bright outer cometary ring + tenuous inner hot dust cloud. Astron. J., 154, 182.

Mathis, J. (1990) Interstellar dust and extinction. Annu. Rev. Astron. Astrophys. 28, 37-70.

McDonald, G., et al. (1996) Production and chemical analysis of cometary ice tholins. Icarus, $122,107-117$.

McNutt Jr., R., et al. (2019) Near-term interstellar probe: First step. Acta Astronautica, 162, 284299.

Moore, M. et al. (2004) Radiation products in processed ices relevant to Edgeworth-Kuiper-Belt Objects. In: Davies, J., Barrera, L. (eds.) The First Decadal Review of the EdgeworthKuiper Belt. Springer.

Poppe, A. (2016) An improved model for interplanetary dust fluxes in the outer Solar System. Icarus, 264, 369-386.

Poppe, A., et al. (2020) The Solar System's Debris Disk. A white paper for the Solar \& Space Physics Decadal Survey 2023.

Reuter, D., et al. (2008) Ralph: A visible/infrared imager for the New Horizons Pluto/Kuiper Belt mission. Space Science Reviews, 140 (1-4), 129-154.

Runyon KBO WP-isp Runyon, K., et al. (2020) Comparative planetology in the Kuiper Belt enabled by an Interstellar Probe. NAS white paper submitted to the Planetary Science decadal survey committee.

Stark, C., Kuchner, M. (2009) A new algorithm for self-consistent three-dimensional modeling of collisions in dusty debris disks. Astrophys. J., 707, 543-553.

Szalay, J., et al. (2020) The interaction of interstellar dust with our heliosphere. NAS white paper submitted to the Astrophysical, Planetary, and Heliophysics Science decadal survey committees.

Tegler, S. (2014) Kuiper Belt Objects: 9.2 Spectroscopy, in: Encyclopedia Solar System (Spohn, T., Breuer, D., Johnson, T., eds.) Third Edition, Elsevier. https://doi.org/10.1016/C20100-67309-3

$\mathrm{Yu}, \mathrm{X}$., et al. (2018) Where does Titan sand come from: Insight from mechanical properties of Titan sand candidates. JGR Planets, 123 (9), 2310-2331. 